\title{
Glycogen: its mode of formation and contribution to hepatic glucose output in postabsorptive humans
}

\author{
W.Pimenta ${ }^{1}$, N. Nurjhan ${ }^{1}$, P.-A. Jansson ${ }^{1}$, M.Stumvoll ${ }^{1}$, J. Gerich ${ }^{1}$, M. Korytkowski ${ }^{2}$ \\ ${ }^{1}$ The Whittier Institute for Diabetes and Endocrinology, La Jolla, California, USA \\ ${ }^{2}$ Department of Medicine, General Clinical Research Center, University of Pittsburgh, Pittsburgh, Pennsylvania, USA
}

\begin{abstract}
Summary To assess the relative contributions of gluconeogenesis and glycogenolysis to overall hepatic glucose output in postabsorptive normal humans and those of the indirect and direct pathways for glycogen synthesis, we studied six normal volunteers, who had been fasted for $16 \mathrm{~h}$ to reduce their hepatic glycogen stores, and then ingested glucose $(250$ g over $10 \mathrm{~h})$ enriched with $\left[6-{ }^{3} \mathrm{H}\right]$ glucose to replenish and label their hepatic glycogen. After a 10 -h overnight fast, release of the $\left[6-{ }^{3} \mathrm{H}\right]$ glucose into the circulation was traced with $\left[2-{ }^{3} \mathrm{H}\right]$ glucose to estimate breakdown of glycogen that had been formed via the direct pathway while gluconeogenesis was simultaneously estimated by incorporation of infused $\left[{ }^{14} \mathrm{C}\right]$ lactate into plasma glucose. We found that release of $\left[6-{ }^{3} \mathrm{H}\right]$ glucose into plasma $\left(6.79 \pm 0.69 \mu \mathrm{mol} \cdot \mathrm{kg}^{-1} \cdot \mathrm{min}^{-1}\right)$ accounted for $46 \pm 5 \%$ of hepatic glucose output $\left(15.0 \pm 0.7 \mu \mathrm{mol} \cdot \mathrm{kg}^{-1}\right.$. $\left.\mathrm{min}^{-1}\right)$ while glucose formed from lactate $(2.71 \pm$ $\left.0.28 \mu \mathrm{mol} \cdot \mathrm{kg}^{-1} \cdot \mathrm{min}^{-1}\right)$ accounted for $19 \pm 2 \%$ of hepatic glucose output. Since these determinations
\end{abstract}

underestimate direct pathway glycogenolysis and overall gluconeogenesis, a maximal estimate for the contribution of indirect pathway glycogenolysis to hepatic glucose output is obtained by subtracting the sum of direct pathway glycogenolysis and lactate gluconeogenesis from hepatic glucose output. This amounted to a maximum of $36 \pm 5 \%$ of hepatic glucose output and $44 \pm 6 \%$ of overall glycogenolysis. Assuming that the relative proportions of direct and indirect pathway glycogen breakdown would reflect the relative contributions of these pathways to glycogen formation, we conclude that under our experimental conditions the direct pathway is the predominant route for glycogen formation in man and that in overnight fasted humans, hepatic glucose output is mainly the result of glycogenolysis. [Diabetologia (1994) 37: 697-702]

Key words Glycogen, gluconeogenesis, glycogenolysis, hepatic glucose output.
In overnight fasted humans, the liver is essentially the only organ supplying glucose to the systemic circulation. It does this via two processes: glycogenolysis (via release of glucose previously stored as glycogen) and gluconeogenesis (via release of glucose newly synthesized from precursors such as lactate, glycerol and amino acids). The relative contributions of these two processes is currently unclear. Splanchnic balance

Received: 23 August 1993

and in revised form: 14 January 1994

Corresponding author: Dr. J.E. Gerich, University of Rochester Medical Center 601 Elmwood Ave, MED/CRC, Rochester NY 14642, USA
$[1,2]$, isotope dilution $[3,4]$ and serial liver biopsy studies [5] have generally indicated that glycogenolysis is the predominant process, accounting for $50-70 \%$ of hepatic glucose output. On the other hand, recent studies using nuclear magnetic resonance to measure the depletion of ${ }^{13} \mathrm{C}$ in hepatic glycogen $[6,7]$ have suggested that gluconeogenesis is the predominant process, accounting for at least two-thirds of hepatic glucose output.

Another currently unresolved issue is the extent to which hepatic glycogen is repleted via the so-called direct and indirect pathways in humans $[8,9]$. With the direct pathway, glycogen is formed from glucose 6phosphate originating from glucose taken up and phosphorylated within the liver and then directly incorpor- 
ated into glycogen whereas with the indirect pathway, glycogen is formed from glucose 6-phosphate originating from gluconeogenic precursors generated either within the liver or extrahepatic tissues $[10,11]$. In vitro studies and experiments in rats have generally indicated that the indirect pathway is the major route for glycogen synthesis [9] but their validity and physiologic significance have been questioned [8]. In humans, estimates for the contribution of the indirect pathway have varied from as little as $25 \%$ to as much as $60 \%$ [12-18].

In the present studies, we employed a novel approach to assess the relative contributions of gluconeogenesis and glycogenolysis to overall hepatic glucose output in postabsorptive normal humans and have also used this approach to assess the relative contributions of the indirect and direct pathways for glycogen synthesis. The approach entails having subjects fast for $16 \mathrm{~h}$ to reduce their hepatic glycogen stores, followed by having them ingest glucose enriched with $\left[6{ }^{3} \mathrm{H}\right]$ glucose to replenish and label their hepatic glycogen; the subsequent release of the $\left[6-{ }^{3} \mathrm{H}\right]$ glucose into the circulation after an overnight fast is traced with $\left[2-{ }^{3} \mathrm{H}\right] \mathrm{glu}-$ cose and provides an index of glycogen breakdown while gluconeogenesis is simultaneously estimated by incorporation of $\left[{ }^{14} \mathrm{C}\right]$ lactate into plasma glucose.

Since gluconeogenic precursors derived from the ingested $\left[6^{3} \mathrm{H}\right]$ glucose lose virtually all their label prior to their incorporation into glycogen [19], this approach measures only breakdown of glycogen formed via the direct pathway. Moreover, this approach provides a minimal estimate of the direct pathway because it uses the specific activity of the ingested glucose rather than specific activity at which glucose is taken up by the liver (arterial and portal venous specific activities) [16]. Nevertheless, our data show that in normal postabsorptive humans, release of glucose from glycogen which had been stored via the direct pathway accounts for nearly $50 \%$ of hepatic glucose output. Thus, under our experimental conditions, glycogenolysis is the predominant process responsible for overall hepatic glucose output in postabsorptive humans and their glycogen is formed mainly via the direct pathway.

\section{Subjects and methods}

Informed written consent was obtained from six (four women, two men) healthy subjects with no family history of diabetes. Their mean ( \pm SEM) age was $45 \pm 2$ years and their mean weight was $75 \pm 7 \mathrm{~kg}$ (body mass index $26.3 \pm 2.3 \mathrm{~kg} / \mathrm{m}^{2}$ ). The protocol was approved by the University of Pittsburgh Biomedical Institutional Review Board.

All subjects were admitted to the inpatient facility of the University of Pittsburgh Clinical Research Center by 10.00 hours on the first day, having fasted since 20.00 hours the night before. The subjects had consumed a weight-maintenance diet containing at least $200 \mathrm{~g}$ carbohydrate for the preceding 3 days. At 11.00 hours a 18 -gauge catheter was inserted into a superficial forearm vein. At 12.00,14.00, 18.00, 20.00 and 22.00 hours, subjects ingested over $5 \mathrm{~min}$ a solution containing
$50 \mathrm{~g}$ of glucose enriched with $25-35 \mu \mathrm{Ci}\left[6-{ }^{3} \mathrm{H}\right]$ glucose (Amersham Int., Amersham Buchs., UK) to replenish and label their hepatic glycogen stores. Otherwise, the subjects ingested nothing but water until completion of the experiments.

At 03.30 hours the next morning, primed $(20 \mu \mathrm{Ci})$ continuous $(0.2 \mu \mathrm{Ci} / \mathrm{min})$ infusions of $\left[2{ }^{3} \mathrm{H}\right]$ glucose (Amersham; for determination of overall hepatic glucose output) and of $\left[3-{ }^{14} \mathrm{C}\right]$ lactate (Amersham; for estimation of glucose formed from lactate, gluconeogenesis) were started. At 07.00 hours a hand vein was cannulated retrogradely with a 20 -gauge intravenous catheter and placed in a thermoregulated box $\left(60^{\circ} \mathrm{F}\right)$ to obtain arterialized venous samples. Thirty min later, (i. e., after a 4-h isotope-equilibration period), two blood samples at approximately 07.30 and 08.00 hours, were collected for determination of plasma glucose and lactate concentrations and for determination of the plasma spcific activities of $\left[{ }^{14} \mathrm{C}\right],\left[2-{ }^{3} \mathrm{H}\right]$, and $\left[6-{ }^{3} \mathrm{H}\right]$ glucose and $\left.{ }^{14} \mathrm{C}\right]$ lactate.

Blood samples for glucose were collected into $\mathrm{NaF}$ oxalate tubes. The tubes were placed on ice and centrifuged at $4^{\circ} \mathrm{C}$. Plasma was separated and stored at $-20^{\circ} \mathrm{C}$ for later analysis. Plasma glucose was determined in duplicate by a glucose oxidase method (YSI glucose analyzer; Yellow Springs Inc., Yellow Springs, Ohio, USA). Lactate was determined microfluorometrically as previously described [15].

Blood for determination of $\left[{ }^{3} \mathrm{H}\right]$ - and $\left[{ }^{14} \mathrm{C}\right]$ glucose and $\left[{ }^{14} \mathrm{C}\right]$ lactate specific activity was immediately deproteinized with an equal volume of chilled $7 \%$ perchloric acid, centrifuged at $4{ }^{\circ} \mathrm{C}$ and the supernatant stored at $-70^{\circ} \mathrm{C}$ for subsequent analysis. Neutralized $(4 \mathrm{~N} \mathrm{KOH})$ aliquots of the perchlorate extracts were applied to stacked anion (AG-1-X8) and cation (AG-50X8) exchange columns (Bio-Rad, Chicago, IIl., USA). The columns were eluted, and glucose and lactate were isolated as previously described [20]. The eluates were evaporated and reconstituted in $1.2 \mathrm{ml}$ distilled $\mathrm{H}_{2} \mathrm{O}$. For lactate, a $0.9-\mathrm{ml}$ aliquot was taken for determination of $\left[{ }^{14} \mathrm{C}\right]$-radioactivity and $0.1 \mathrm{ml}$ was used for determination of lactate concentration. For glucose, $0.2 \mathrm{ml}$ was taken for determination of glucose concentration. Another $0.5-\mathrm{ml}$ aliquot was used for determination of total $\left[{ }^{3} \mathrm{H}\right]$ and $\left[{ }^{14} \mathrm{C}\right]$ radioactivity and another $0.5 \mathrm{ml}$ was used to determine the $\left[{ }^{3} \mathrm{H}\right]$ and $\left[{ }^{14} \mathrm{C}\right]$ in carbon 6 of glucose according to the method of Bloom [21]. Adjustment for efficiency of the degradation and subsequent dimedone formation was made by concurrently using recovery of ${ }^{14} \mathrm{C}$ on $\mathrm{C}-6$ of glucose using external standards. $\left[2{ }^{3} \mathrm{H}\right]$ Glucose radioactivity was calculated as the difference between total $\left[{ }^{3} \mathrm{H}\right]$ radioactivity and $\left[6-{ }^{3} \mathrm{H}\right]$ glucose radioactivity. Using this approach, less than $0.4 \%$ of the radioactivity in $\left[2{ }^{3} \mathrm{H}\right]$ glucose and more than $99 \%$ of the radioactivity in $\left[6-{ }^{3} \mathrm{H}\right]$ glucose is recovered in the dimedone with coefficients of variation less than $4 \%$.

The rates of overall glucose appearance, i.e. hepatic glucose output lactate appearance and rates of $\left[{ }^{14} \mathrm{C}\right]$ glucose appearance, an index of lactate gluconeogenesis, were calculated using steady-state equations [20]. Rates of appearance of $\left[6-{ }^{3} \mathrm{H}\right]$ glucose, an index of glycogenolysis were calculated using the equation of Chiasson [22].

Unless otherwise indicated, data in text are given as means \pm SEM. Hepatic glucose output attributable to breakdown of glycogen formed by the direct pathway was calculated by dividing the rate of appearance of $\left[6-{ }^{3} \mathrm{H}\right]$ glucose $\left(\mathrm{dpm} \cdot \mathrm{kg}^{-1} \cdot \mathrm{min}^{-1}\right)$ by the specific activity of the ingested glucose [16]. A maximal estimate of hepatic glucose output attributable to breakdown of glycogen formed by the indirect pathway was calculated as the difference between overall hepatic glucose output and the sum of direct pathway glycogenolysis and lactate gluconeogenesis. 
Table 1. Specific activities (SA) of plasma $\left[2-{ }^{3} \mathrm{H}\right],\left[6-{ }^{3} \mathrm{H}\right],\left[{ }^{14} \mathrm{C}\right]$ glucose and $\left[{ }^{14} \mathrm{C}\right]$ lactate

\begin{tabular}{|c|c|c|c|c|c|c|}
\hline \multirow[b]{2}{*}{ Subject } & \multicolumn{3}{|c|}{$\begin{array}{l}{\left[2-{ }^{3} \mathrm{H}\right] \text { glucose } \mathrm{SA}} \\
(\mathrm{dpm} / \mu \mathrm{mol})\end{array}$} & \multicolumn{3}{|c|}{$\begin{array}{l}{\left[6-{ }^{3} \mathrm{H}\right] \text { glucose } \mathrm{SA}} \\
(\mathrm{dpm} / \mu \mathrm{mol})\end{array}$} \\
\hline & $240 \mathrm{~min}$ & $270 \mathrm{~min}$ & average & $240 \mathrm{~min}$ & $270 \mathrm{~min}$ & average \\
\hline 1 & 555 & 477 & 516 & 107 & 131 & 119 \\
\hline 2 & 448 & 428 & 438 & 142 & 150 & 146 \\
\hline 3 & 605 & 701 & 653 & 49 & 39 & 44 \\
\hline 4 & 337 & 305 & 321 & 133 & 126 & 130 \\
\hline 5 & 331 & 300 & 316 & 135 & 144 & 140 \\
\hline 6 & 288 & 291 & 290 & 127 & 143 & 135 \\
\hline mean & 427 & 217 & 422 & 116 & 122 & 119 \\
\hline \multirow[t]{3}{*}{$\pm \mathrm{SEM}$} & 49 & 59 & 53 & 13 & 16 & 14 \\
\hline & \multicolumn{3}{|c|}{$\begin{array}{l}{\left[{ }^{14} \mathrm{C}\right] \text { lactate SA }} \\
(\mathrm{dpm} / \mu \mathrm{mol})\end{array}$} & \multicolumn{3}{|c|}{$\begin{array}{l}{\left[{ }^{14} \mathrm{C}\right] \text { glucose } \mathrm{SA}} \\
(\mathrm{dpm} / \mu \mathrm{mol})\end{array}$} \\
\hline & $240 \mathrm{~min}$ & $270 \mathrm{~min}$ & average & $240 \mathrm{~min}$ & $270 \mathrm{~min}$ & average \\
\hline 1 & 585 & 556 & 571 & 113 & 116 & 115 \\
\hline 2 & 339 & 341 & 340 & 81 & 85 & 83 \\
\hline 3 & 618 & 669 & 644 & 325 & 326 & 326 \\
\hline 4 & 311 & 299 & 305 & 163 & 155 & 159 \\
\hline 5 & 331 & 340 & 336 & 112 & 135 & 124 \\
\hline 6 & 359 & 343 & 351 & 136 & 138 & 137 \\
\hline mean & 424 & 425 & 424 & 155 & 159 & 157 \\
\hline \pm SEM & 52 & 56 & 54 & 33 & 32 & 32 \\
\hline
\end{tabular}

Table 2. Rates of hepatic glucose output, lactate gluconeogenesis, and direct pathway glycogenolysis

\begin{tabular}{llll}
\hline Subject & $\begin{array}{l}\text { Hepatic glucose } \\
\text { output }^{\mathrm{a}}\end{array}$ & $\begin{array}{l}\text { Glucose formed } \\
\text { from lactate }^{\mathrm{a}}\end{array}$ & $\begin{array}{l}\text { Direct pathway } \\
\text { glycogenolysis }^{\mathrm{a}}\end{array}$ \\
\hline 1 & 15.5 & 1.55 & 6.22 \\
2 & 17.1 & 2.08 & 9.42 \\
3 & 11.7 & 2.97 & 7.26 \\
4 & 13.7 & 3.58 & 4.37 \\
5 & 15.4 & 2.83 & 8.08 \\
6 & 16.7 & 3.25 & 5.38 \\
mean & 15.0 & 2.71 & 6.79 \\
\pm SEM & 0.7 & 0.28 & 0.69 \\
\hline
\end{tabular}

${ }^{\mathrm{a}} \mu \mathrm{mol} \cdot \mathrm{kg}^{-1} \cdot \mathrm{min}^{-1}$

\section{Results}

Plasma glucose and lactate concentrations averaged $5.20 \pm 0.13$ and $0.95 \pm 0.10 \mathrm{mmol} / 1$, respectively, $10 \mathrm{~h}$ after the final glucose ingestion, i.e., 08.00 hours. Mean plasma-specific activities of $\left[2-{ }^{3} \mathrm{H}\right],\left[6-{ }^{3} \mathrm{H}\right],\left[{ }^{14} \mathrm{C}\right]$ glucose and $\left[{ }^{14} \mathrm{C}\right]$ lactate after $4 \mathrm{~h}$ of isotope infusions (07.30 hours) differed by less than $5 \%$ from those after $4.5 \mathrm{~h}$ of isotope infusions ( 08.00 hours) with no consistent changes among individual values (Table 1 ). These observations thus suggest that by $10 \mathrm{~h}$ after the final glucose ingestion and by $4 \mathrm{~h}$ after initiation of $\left[2-{ }^{3} \mathrm{H}\right]$ glucose and $\left[{ }^{14} \mathrm{C}\right]$ lactate infusions, isotopic steady state had been approximated.

Rates of overall glucose appearance in plasma (i.e., hepatic glucose output), appearance of glucose in plasma formed from lactate, and appearance of glucose released into plasma from glycogen formed via the direct pathway (Table 2) averaged $15.0 \pm 0.7,2.71 \pm 0.28$ and $6.79 \pm 0.69 \mu \mathrm{mol} \cdot \mathrm{kg}^{-1} \cdot \mathrm{min}^{-1}$, respectively. Gluconeo- genesis from lactate and direct pathway glycogenolysis accounted for $18.6 \pm 2.4$ and $45.7 \pm 4.7 \%$ of overall hepatic glucose output, respectively. A maximal estimate for the contribution of indirect pathway glycogenolysis to hepatic glucose output can be calculated if one assumes that glucose formed from lactate represented all of gluconeogenesis and that there were no underestimation of direct pathway glycogenolysis; this would yield a maximum of $35.7 \pm 5.2 \%$ of hepatic glucose output that could be accounted for by indirect pathway glycogenolysis. Furthermore, if one assumes that the relative rate of release of glucose from the liver via direct and indirect pathway glycogenolysis reflects the relative rate of synthesis of glycogen via these pathways, then a maximum of $43.8 \%$, i. e. $[35.7 /(35.7+45.7)]$, of glycogen repletion could occur via the indirect pathway under our experimental conditions.

\section{Discussion}

Various methodologic limitations have made it difficult to obtain precise estimates in humans of the contributions of gluconeogenesis and glycogenolysis to hepatic glucose output and of the pathways used for repletion of hepatic glycogen. As a consequence, estimates of the contribution of gluconeogenesis to hepatic glucose output after an overnight fast have ranged from 35 to $70 \%$ [1-7] and estimates of the contribution of the indirect pathway for glycogen repletion have ranged from 25 to $80 \%$ [12-18].

In the present studies we have attempted to better define the contribution of these processes by simultaneously obtaining minimal estimates for direct pathway glycogenolysis and gluconeogenesis and a maximal estimate of indirect pathway glycogen repletion. Our approach entailed having subjects fast for $16 \mathrm{~h}$ to reduce their hepatic glycogen stores, followed by having them ingest glucose enriched with $\left[6-{ }^{3} \mathrm{H}\right]$ glucose to replenish and label their hepatic glycogen; the subsequent release of the $\left[6{ }^{3} \mathrm{H}\right]$ glucose into the circulation after an overnight fast was traced with $\left[2-{ }^{3} \mathrm{H}\right]$ glucose to provide an index of the release of glucose from glycogen formed via the direct pathway while release of $\left[{ }^{14} \mathrm{C}\right]$ glucose formed from $\left[{ }^{14} \mathrm{C}\right]$ lactate was used as an index of gluconeogenesis. It was assumed that glucose released from glycogen via direct and indirect pathway glycogenolysis would reflect the relative contribution of these pathways to the repletion of hepatic glycogen and thus the maximal contribution of the indirect pathway was calculated as the difference between overall hepatic glucose output and the sum of direct pathway glycogenolysis and gluconeogenesis from lactate.

Using this approach, we found that direct pathway glycogenolysis accounted for a minimum of $45.7 \pm 4.7 \%$ of hepatic glucose output while gluconeogenesis from lactate accounted for a minimum of $18.6 \pm 2.4 \%$, leaving a maximum of $35.7 \pm 5.2 \%$ for in- 
direct pathway glycogenolysis. Assuming that the relative release of glucose via direct and indirect pathway glycogenolysis would reflect the relative contributions of these pathways for glycogen repletion, these data indicate that the indirect pathway could be responsible for a maximum of $43.8 \%$, i.e. $[35.7 /(35.7+45.7)]$ of overall hepatic glycogen repletion. Thus, under our experimental conditions, glycogenolysis was the predominant process responsible for hepatic glucose output and the direct pathway was the major route for hepatic glycogen repletion.

Certain potentially important limitations of the present approach deserve discussion. First of all, we used $\left[2-{ }^{3} \mathrm{H}\right]$ glucose to estimate overall hepatic glucose output. This was done because $\left[2-{ }^{3} \mathrm{H}\right]$ glucose, unlike [6- or $\left.3^{3} \mathrm{H}\right]$ glucose, is not incorporated into glycogen. As pointed out by Radziuk [16], use of [ 6 or $\left.3{ }^{3} \mathrm{H}\right] \mathrm{glu}-$ cose would have been inappropriate, because, under our experimental conditions, it could have led to an underestimation of hepatic glucose output due to release of $\left[{ }^{3} \mathrm{H}\right]$ glucose from glycogen, which would diminish dilution of plasma $\left[{ }^{3} \mathrm{H}\right]$ glucose specific activity (i.e. labelled and unlabelled glucose would be released into plasma). On the other hand, a drawback of using $\left[2-{ }^{3} \mathrm{H}\right]$ glucose is that it normally overestimates hepatic glucose output by $10-20 \%$ due to its detritiation and subsequent release into plasma after glucose-glucose 6phosphate cycling [23]. It should be pointed out, however, that this slight overestimate of hepatic glucose output would introduce comparable errors in our estimates of glycogenolysis and gluconeogenesis, since the value for hepatic glucose output is used in the equation to calculate both processes. Therefore, overestimation of hepatic glucose output due to use of $\left[2-{ }^{3} \mathrm{H}\right]$ glucose as tracer should not have substantially affected our conclusions regarding the relative contribution of these processes to hepatic glucose output.

Secondly, in our approach, the division of the appearance of $\left[6-{ }^{3} \mathrm{H}\right]$ glucose in plasma by the ingested $\left[6-{ }^{3} \mathrm{H}\right]$ glucose specific activity to convert it from $\mathrm{dpm} \cdot \mathrm{kg}^{-1} \cdot \mathrm{min}^{-1}$ to $\mathrm{mg} \cdot \mathrm{kg}^{-1} \cdot \mathrm{min}^{-1}$ leads to an underestimation of the release of glucose from glycogen formed by the direct pathway. This follows from the fact that during absorption of $\left[6-{ }^{3} \mathrm{H}\right]$ glucose into plasma, its specific activity is diluted by unlabelled glucose in plasma. Consequently, the specific activity at which glucose is taken up by the liver and converted to glycogen (i.e., hepatic arterial and portal vein specific activities) is lower than that of the ingested glucose [16]. Our own data [24] and those of Radziuk [16] indicate that arterial specific activites are $10-20 \%$ lower than those of the ingested glucose, indicating an underestimation of direct pathway glycogenolysis by $10-20 \%$. Correction for this would increase the contribution of direct pathway glycogenolysis to $50-55 \%$ of hepatic glucose output. In retrospect a more accurate estimate would have been obtained had we measured and used the mean arterial $\left[6{ }^{3} \mathrm{H}\right]$ glucose specific activity during which glucogen was being repleted.

Thirdly, the present approach assumes that all of the ingested glucose had been absorbed and removed from plasma so that at the time of the measurements $(10 \mathrm{~h}$ after the final glucose ingestion), the only source of plasma $\left[6-{ }^{3} \mathrm{H}\right]$ glucose is breakdown of glycogen. Regarding this assumption, the studies of Radziuk [14] and Jackson et al. [25] indicate that absorption of ingested glucose would be complete by $4.5-5 \mathrm{~h}$. Furthermore, the data of Jackson et al. [25], Mitrakou et al. [10], and Kelley et al. [24] indicate that at least $80 \%$ of the ingested glucose would have been removed from plasma within $5 \mathrm{~h}$ of its ingestion. Given a half-life in plasma of $44.4 \mathrm{~min}$ for glucose [25], it can be calculated that less than $0.15 \%$ of the last ingested glucose load would not have been removed from plasma. Thus, the assumption that all of the $\left[6-{ }^{3} \mathrm{H}\right]$ glucose in plasma at the time of our measurements originated solely from breakdown of glycogen seems to be justified.

Finally, the present approach assumes that glycogen release occurs as a last on, first off process and that glycogen is laid down at a uniform specific activity. The validity of the first assumption is supported by the studies of Devos and Hers [26] and the branching structure of glycogen in which additional glucose moieties are added to peripheral branches and degradation proceeds centripedally. Regarding the second assumption, the studies of Mitrakou et al. [10] and Kelley et al. [24] indicate that hepatic glucose output is maximally suppressed for at least $3 \mathrm{~h}$ after glucose is ingested. Thus, ingestion of glucose at 2 -h intervals should have resulted in deposition of glycogen at a near uniform specific activity.

Our results indicating direct pathway glycogenolysis alone accounts for a minimum of $46 \%$ of overall hepatic glucose output thus provide strong evidence that glycogenolysis is the predominant process responsible for hepatic glucose output in overnight fasted humans and are consistent with estimates based on previous isotope dilution $[3,4]$, splanchnic balance $[1,2]$ and serial liver biopsy [5] studies which generally indicated that glycogenolysis accounted for more than $60 \%$ of hepatic glucose output in overnight fasted humans.

Our results are, however, at variance with values recently reported using $\mathrm{C}^{13}$ nuclear magnetic resonance to measure glycogen depletion $[6,7]$. In these studies, it was found that after a $23-\mathrm{h}$ fast, glycogenolysis accounted for $30 \%$ [7] and $35 \%$ [6] of overall hepatic glucose output in normal volunteers. Certain methodologic differences may explain this variance. First of all, our subjects were studied after a 10 -h fast. Secondly, our subjects received more carbohydrate prior to beginning their fast $(250 \mathrm{~g})$ than the subjects in the $\mathrm{C}^{13}$ nuclear magnetic resonance studies $(\sim 100 \mathrm{~g})$. Thirdly, in the latter studies $[6,7]$ rates of glycogenolysis prior to the 23-h fast were extrapolated using linear regression since liver size was measured only at 0.5 and 
$67.5 \mathrm{~h}$ of the fast and rates of hepatic glucose production were not measured before $23 \mathrm{~h}$ of the fast. Nevertheless, it should be pointed out that our results indicating glycogenolysis could account for more than $70 \%$ of hepatic glucose output after a 10 -h overnight fast would not be inconsistent with glycogenolysis accounting for only $30-35 \%$ of hepatic glucose output after a 23 -h fast.

Assuming that the pattern of release of glucose into plasma from glycogen would reflect the pathways by which it was stored, one can derive a maximal estimate of the contribution of indirect pathway glycogen formation by subtracting from overall hepatic glucose output, the contribution of glucose derived from direct pathway glycogenolysis and the contribution of glucose due to ongoing gluconeogenesis. We found that the indirect pathway could account for at most $44 \%$ hepatic glycogen repletion. Since we measured only gluconeogenesis from lactate and we underestimated direct pathway glycogenolysis by $10-20 \%$, it can be readily appreciated that, under our experimental conditions, the indirect pathway was not the major route for hepatic glycogen repletion, probably accounting for only about a third.

Finally, one needs to consider the physiologic relevance of our method of replenishing hepatic glycogen stores; it is possible that our having subjects ingest $50 \mathrm{~g}$ glucose five times over a 10 -h period might have selectively promoted use of the direct pathway since Shulman et al. [18] found greater direct pathway glycogen formation in humans after having eaten breakfast than after having fasted overnight. We believe that our mode of hepatic glycogen repletion would not be very different from the daily eating habits of many people, since lunch, dinner and a bedtime snack could readily lead to consumption of $250 \mathrm{~g}$ carbohydrate over a $10-\mathrm{h}$ period. Moreover, our estimates of the contributions of the direct and indirect pathways to glycogen repletion are consistent with several previous reports examining this issue under different experimental conditions in humans $[12-15,18]$.

These estimates $[12-15,18]$ are, however, at variance with the estimates of about $60 \%$ of glycogen formation occurring via the indirect pathway based on incorporation of $\left[1-{ }^{14} \mathrm{C}\right]$ glucose and $\left[{ }^{14} \mathrm{CO}_{2}\right]$ into glycogen during ingestion of a glucose load and the subsequent release into plasma of $\left[{ }^{14} \mathrm{C}\right]$ glucose by infusion of glucagon $[16,17]$. In the study in which $\left[1-{ }^{14} \mathrm{C}\right]$ glucose was used, $\left[1-{ }^{14} \mathrm{C}\right]$ glucose released into plasma by the glucagon infusion accounted for approximately $73 \%$ of all the $\left[{ }^{14} \mathrm{C}\right]$ glucose released into plasma. If the correction factor for $\left[3-{ }^{14} \mathrm{C}\right]$ lactate Krebs cycle carbon exchange is as low as reported by Magnusson et al. [27] in fed humans, then these results would be comparable to ours. In the studies using ${ }^{14} \mathrm{CO}_{2}$, Krebs cycle carbon exchange was not measured but a correction factor of 3.3 was used based on studies in fasted dogs. This may not be valid for humans [27].
In summary, our results indicate that, under our experimental conditions, in 10 -h overnight fasted normal humans, glycogenolysis accounts for the majority of hepatic glucose output and that the direct pathway is the predominant route for repletion of hepatic glycogen in humans.

Acknowledgements. We thank the staff of the General Clinical Research Center, the research participants, and Ms. D.Purdy, Ms. C. Korbanic, Mr. D. Kirsh and Ms. D. McMillen for their excellent technical help. The expert editorial assistance of Ms. L. Brinker is also acknowledged. This work was supported in part by funds from the National Institutes of Health DRR/GCRC (M501, RR00056) and the DEMD/NDDDK (DK20411), Brazilian Grant \# CNPq \# 201215/90.0 and generous donors to the Whittier Institute.

\section{References}

1. Dietze G, Wicklmayr M, Hepp K, et al (1976) On gluconeogenesis of human liver: accelerated hepatic glucose formation by increased precursor supply. Diabetologia 12: 555-561

2. Wahren J, Felig P, Cerasi E, Luft R (1972) Splanchnic and peripheral glucose and amino acid metabolism in diabetes mellitus. J Clin Invest 51: 1870-1878

3. Consoli A, Kennedy F, Miles J, Gerich J (1987) Determination of Krebs cycle metabolic carbon exchange in vivo and its use to estimate gluconeogenesis in man. J Clin Invest 80: $1303-1310$

4. Consoli A, Nurjhan N, Capani F, Gerich J (1989) Predominant role fo gluconeogenesis in increased hepatic glucose production in NIDDM. Diabetes 38: 550-561

5. Nilsson L, Hultman E (1973) Liver glycogen in man - the effect of total starvation or a carbohydrate-poor diet followed by refeeding. Scand J Lab Invest 32: 325-330

6. Rothman D, Magnusson I, Katz L, Shulman R, Shulman G (1991) Quantitation of hepatic glycogenolysis and gluconeogenesis in fasting humans with 13C NMR. Science 254: 573 575

7. Magnusson I, Rothman D, Katz L, Shulman R, Shulman G (1992) Increased rate of gluconeogenesis in type II diabetes. A ${ }^{13} \mathrm{C}$ nuclear magnetic resonance study. J Clin Invest 90 : 1323-1327

8. Shulman G, Landau B (1992) Pathways of glycogen repletion. Physiol Rev 72: 1019-1035

9. McGarry J, Kuwajima M, Newgard C, Forster D, Katz J (1987) From dietary glucose to hepatic glycogen: the full circle round. Ann Rev Nutr 7: 51-73

10. Mitrakou A, Jones R, Okuda Y, et al (1991) Pathway and carbon sources for hepatic glycogen repletion in the dog. Am J Physiol 260: E194-E202

11. Moore M, Cherrington A, Cline G, et al (1991) Source of car bons for hepatic glycogen synthesis in the conscious dog. J Clin Invest 88: 578-587

12. Magnusson I, Chandramouli V, Schumann W, Kumaran K, Wahren J, Landau B (1987) Quantitation of the pathways of hepatic glycogen formation on ingesting a glucose load. $\mathrm{J}$ Clin Invest 80: 1748-1754

13. Magnusson I, Chandramouli V, Schumann W, Kumaran K, Wahren J, Landau B (1989) Pathways of hepatic glycogen formation in humans following ingestion of a glucose load in the fed state. Metabolism 38: 583-585

14. Radziuk J (1982) Sources of carbon in hepatic glycogen synthesis during absorption of an oral glucose load in humans. Fed Proc 41: 111-117 
15. Consoli A, Nurjhan N, Gerich J (1989) Rate of appearance and disappearance of plasma lactate after oral glucose: implications for indirect-direct pathway hepatic glycogen repletion in man. Clin Physiol Biochem 7: 70-78

16. Radziuk J (1989) Hepatic glycogen in humans. I. direct formation after oral and intravenous glucose or after a $24-\mathrm{h}$ fast. Am J Physiol 257: E145-E157

17. Radziuk J (1989) Hepatic glycogen in humans. II. Gluconeogenetic formation after oral and intravenous glucose. Am J Physiol 257: E158-E169

18. Shulman G, Cline G, Schumann W, Chandramouli V, Kumaran K, Landau B (1990) Quantitative comparison of pathways of glycogen repletion in fed and fasted humans. Am J Physiol 259: E335-341

19. Wajgnot A, Chandramouli V, Schumann W, Kumarin K, Efendic S, Landau B (1989) Test of the assumptions made in estimating the extent of futile cycling. Am J Physiol 256: E668-E675

20. Kreisberg RA, Pennington LF, Boshell BR (1970) Lactate turnover and gluconeogenesis in normal and obese humans. Diabetes 19: 53-63

21. Bloom B (1962) The simultaneous determination of $\mathrm{C}^{14}$ and $\mathrm{H}^{3}$ in the terminal groups of glucose. Anal Biochem 3: 85-87
22. Chiasson JL, Liljenquist JE, Lacy WW, Jennings AS, Cherrington AD (1977) Gluconeogenesis: methodological approaches in vivo. Fed Proc 36: 229-235

23. Bell P, Firth R, Rizza R (1989) Assessment of the postprandial pattern of glucose metabolism in nondiabetic subjects and patients with noninsulin-dependent diabetes mellitus using a simultaneous infusion of [2-3H] and [3-3H]- glucose. Metabolism 38: 38-45

24. Kelley D, Mitrakou A, Marsh H, et al (1988) Skeletal muscle glycolysis, oxidation, and storage of an oral glucose load. J Clin Invest 81: 1563-1571

25. Jackson RA, Roshania RD, Hawa MI, Sim BM, DiSilvio L (1986) Impact of glucose ingestion on hepatic and peripheral glucose metabolism in man: an analysis based on simultaneous use of the forearm and double isotope technique. J Clin Endocrinol Metab 63: 541-549

26. Devos P, Hers H (1979) A molecular order in the synthesis and degradation of glycogen in the liver. Eur J Biochem 99: 161-167

27. Magnusson I, Schumann W, Bartsch G, et al (1991) Noninvasive tracing of Krebs cycle metabolism in liver. $\mathbf{J}$ Biochem 266: $6975-6984$ 